\title{
BMJ Open Efficacy and safety of rivaroxaban in patients with inferior vena cava filter placement without anticoagulation contraindications (EPICT): a prospective randomised controlled trial study protocol
}

Libin Zhang, ${ }^{1}$ Miaomiao Li, ${ }^{1}$ Yuefeng Zhu, ${ }^{2}$ Zhenyu Shi, ${ }^{3}$ Wan Zhang, ${ }^{4}$ Bin Gao, ${ }^{5}$ Lubin Li, ${ }^{6}$ Zhengdong Fang, ${ }^{7}$ Guangwei Yang, ${ }^{8}$ Wei Han, ${ }^{9}$ Linjun Wang, ${ }^{10}$ Li Yin, ${ }^{1}$ Xueying $\mathrm{Ke},{ }^{2}$ Jianing Yue, ${ }^{3}$ Zheng Gu, ${ }^{11}$ Zhenjie Liu (D) ${ }^{1}$

To cite: Zhang L, Li M, Zhu Y, et al. Efficacy and safety of rivaroxaban in patients with inferior vena cava filter placement without anticoagulation contraindications (EPICT): a prospective randomised controlled trial study protocol. BMJ Open 2021;11:e045530. doi:10.1136/ bmjopen-2020-045530

- Prepublication history and additional supplemental material for this paper are available online. To view these files, please visit the journal online (http://dx.doi.org/10.1136/ bmjopen-2020-045530).

Received 21 January 2021 Accepted 11 September 2021

Check for updates

(c) Author(s) (or their employer(s)) 2021. Re-use permitted under CC BY-NC. No commercial re-use. See rights and permissions. Published by BMJ.

For numbered affiliations see end of article.

Correspondence to

Zhenjie Liu;

lawson4001@zju.edu.cn

\begin{abstract}
Introduction Inferior vena cava (IVC) filters are commonly used in patients with venous thromboembolism to prevent fatal pulmonary embolism, but the thrombosis risk increases after filter placement. Warfarin is a widely anticoagulant, but long-term monitoring and dose adjustments are required. Anticoagulation with rivaroxaban is more straightforward as it dose not require laboratory monitoring. This study compares the efficacy and safety of rivaroxaban and warfarin as an in anticoagulation therapy for patients with IVC filter placement.

Methods and analysis This is a multicentre, randomised controlled trial. In total, 200 patients with deep vein thrombosis (DVT) with IVC filter implantation from 10 hospitals will be recruited. The patients will be randomised to the experimental group (rivaroxaban) or the control group (nadroparin overlapped with warfarin). The primary outcomes include death of any cause, pulmonary embolism (PE)-related death, bleeding and recurrent PE/ DVT. The secondary outcomes include the percentage of other vascular events, IVC filter retrieval failure and net clinical benefits. This study aims to provide reliable, verification for the efficacy and safety of rivaroxaban antithrombotic therapy after IVC filter placement. Ethics and dissemination The study was approved by the Human Research Ethics Committee of the Second Affiliated Hospital of Zhejiang University School of Medicine (approval number: (2019) 295). The results will be disseminated through presentations at scientific conferences and publications in peer-reviewed journals Trial registration number NCT04066764.
\end{abstract}

\section{INTRODUCTION}

Venous thromboembolism (VTE), including deep vein thrombosis (DVT) and pulmonary embolism (PE), is a common and potentially fatal disease with an annual incidence of approximately one or two cases per 1000 persons in the general population, which
Strengths and limitations of this study

- This study is a prospective, randomised, multicentre, controlled trial.

- This is the first trial to compare the safety and efficacy of rivaroxaban and vitamin $\mathrm{K}$ antagonists in patients with inferior vena cava filter placement.

- This is an open-label study, and researchers and competent physicians are aware of the patients' grouping information.

- Patients in the control (ie, warfarin) group require international normalised ratio and dose adjustment monitoring during the follow-up period, so they are not blinded to the treatment strategy, resulting in bias.

- The participating centres have different brands of retrieval inferior vena cava filters because of variable medical insurance policies, leading to potential errors in the results.

is mostly observed in patients older than 55 years. $^{12}$ The standard VTE treatment is anticoagulation, which effectively stops the abnormal clotting process, preventing DVT, $\mathrm{PE}$ and recurrent VTE progression. ${ }^{3}$ However, inferior vena cava (IVC) filter placement is considered for patients with a recent proximal DVT who show contraindication to therapeutic anticoagulation during acute treatment. Filters are also placed in patients with suspected recurrent VTE or progressive DVT despite therapeutic anticoagulation, extensive DVT (involving the vena cava or iliac veins) or DVT with a free-floating proximal end, proximal DVT in patients undergoing a catheter-directed thrombus reduction procedure or PE managed with thrombolysis or 
surgical embolectomy ${ }^{4-7}$ IVC filters also have prophylactic indications, such as when applied in trauma patients or before bariatric and pelvis surgeries. ${ }^{4}$ Changes in the IVC haemodynamics after filter implantation may increase the risk of thrombosis in or around the filter, and the thrombosis risk significantly increases once the filter is tilted. ${ }^{8}$ For patients with an IVC filter and without anticoagulation contraindications, adequate anticoagulation therapy inhibits the spread of thrombus and promotes autolysis, preventing filter-related thrombosis and PE recurrence. ${ }^{69}$

Until recently, the standard treatment for acute VTE was initial parenteral heparin (eg, low-molecular-weight heparin) administration with overlapping vitamin $\mathrm{K}$ antagonist (VKA) administration, with a target international normalised ratio (INR) of 2.0-3.0. ${ }^{3}{ }^{10}$ Studies have confirmed that low-molecular-weight heparin is safe and effective in the prophylaxis and initial treatment of VTE, ${ }^{11} 12$ and is suitable for cancer and pregnant patients. ${ }^{13-15}$ Warfarin is a standard oral anticoagulant with a sufficient anticoagulant effect and low price. The efficacy and safety of anticoagulation using lowmolecular-weight heparin overlapped with adjusted doses of VKAs in patients with IVC filter implantation are widely recognised. ${ }^{16}$ However, VKA treatment requires laboratory monitoring and dose adjustment and may be complicated by drug and food interactions, presenting a challenge for outpatient management. Altogether, follow-up management is more difficult, and there is more uncertainty regarding the treatment effect and potential for more severe bleeding complications. ${ }^{17}$ The annual major bleeding risk associated with VKAs is 1\%-2\% after the first year. ${ }^{3}$ Non-adherence to warfarin therapy during VTE treatment has also been associated with increased risk for recurrent VTE events. ${ }^{18}$

Rivaroxaban, an oral direct factor Xa inhibitor, has several advantages over VKAs, including rapid onset and predictable pharmacokinetic profile, allowing for simplified drug administration in a standardised dose, and avoiding the need for laboratory monitoring and dose adjustments. There are also no food interactions and only a few drug interactions. ${ }^{119}$ Previous studies have shown that rivaroxaban prevents DVT after orthopaedic surgery, ${ }^{19-21}$ offering an effective, safe, single-drug approach to the initial and continued treatment of DVT and $\mathrm{PE},{ }^{19} 22$ even in patients with active cancer. ${ }^{23} 24$

There are limited clinical data on the safety and effectiveness of rivaroxaban as an anticoagulant in patients with an IVC filter. Therefore, we are conducting a prospective, randomised, multicentre, controlled trial to assess the efficacy and safety of rivaroxaban for preventing DVT in patients with an IVC filter. This study aims to provide the basis for VTE treatment guidelines and explore the clinical indications for rivaroxaban.

\section{METHODS AND ANALYSIS}

\section{Study design and setting}

The study is a randomised, multicentre, open-label, controlled trial comparing the efficacy and safety of

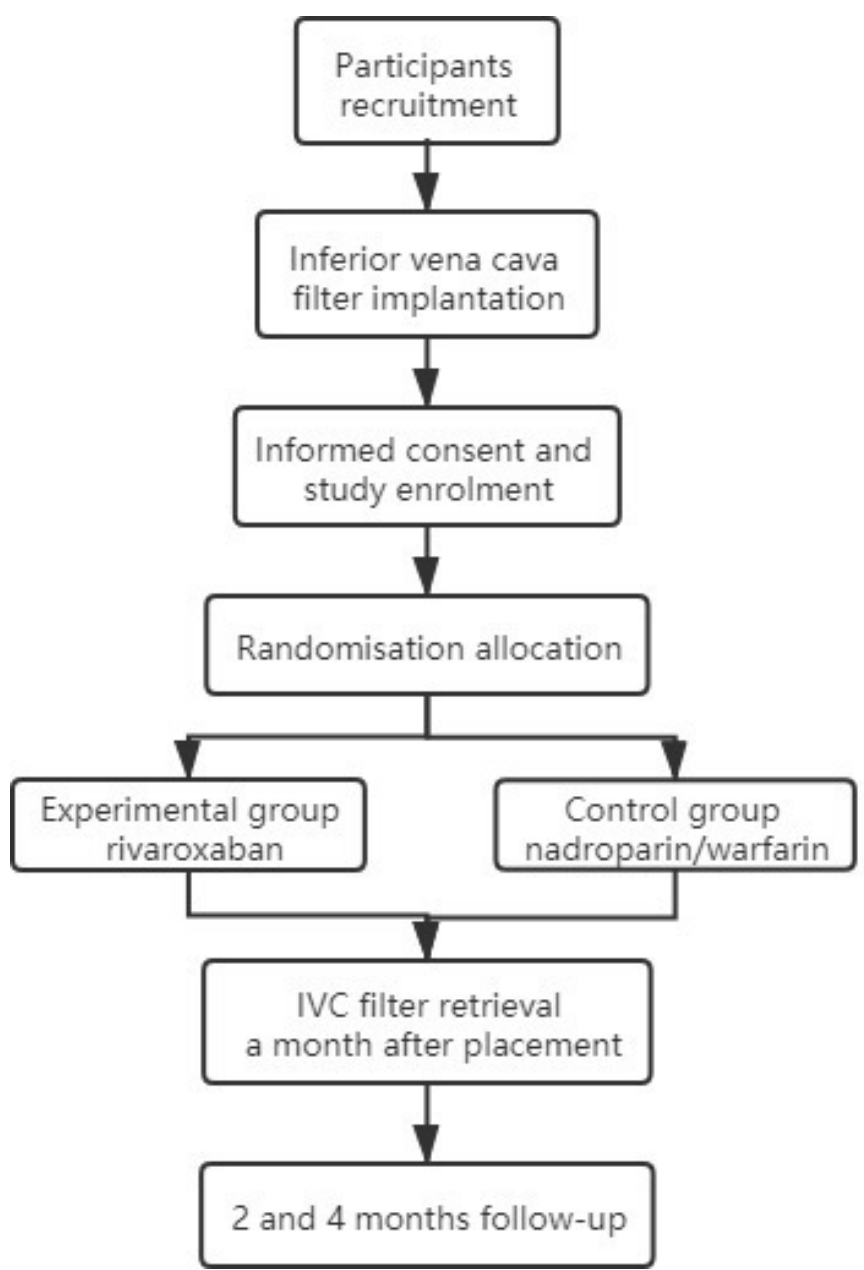

Figure 1 The flow of screening, randomisation, treatment and follow-up of patients in the trial. IVC, inferior vena cava

rivaroxaban with standard therapy consisting of enoxaparin and a VKA in patients with IVC filter placement. The ethics committee approved the study at each participating hospital. Written informed consent was obtained from the patients before randomisation. The data will be collected and maintained by the sponsor. All suspected outcome events will be classified by a central adjudication committee whose members are unaware of the treatment assignments. An independent data and safety monitoring board will periodically review the outcomes. Patients are randomly allocated to either the experimental or control group after enrolment. Figure 1 illustrates the study design.

\section{Sites and patients}

In total, 10 hospitals attended the kick-off meeting and discussed the research protocol and details. All 10 hospitals fully communicated and discussed the DVT clinical diagnosis and treatment procedure, indications for IVC filter placement and the optimal retrieval window according to the existing clinical guidelines. Moreover, the anticoagulation treatment and monitoring programmes after IVC filter implantation were formulated. The diagnosis and treatment process, diagnostic 
criteria and research protocols were approved according to the revised discussion results and the agreement of all participating units.

All patients aged 18-75 years, diagnosed with DVT of the lower extremity and implanted with an IVC filter from the 10 participating hospitals will be recruited. Patients with typical symptoms will be screened through D-dimer testing, colour Doppler ultrasound, CT venography, magnetic resonance venography or venography with digital subtraction angiography to objectively confirm DVT. The indications for IVC filter placement were: ${ }^{3525}$ (1) DVT or PE with contraindication to anticoagulation, (2) recurrent VTE or progressive DVT despite therapeutic anticoagulation, (3) large free-floating proximal DVT in the vena cava or iliac veins, (4) prior to catheter-directed thrombolysis, percutaneous mechanical thrombectomy or surgical embolectomy, (5) DVT of the lower limb with ipsilateral limb surgery and (6) prophylactic indications, such as in trauma patients or before bariatric, pelvis or lower limb surgeries.

Patients conforming to the inclusion and exclusion criteria will be randomised. The inclusion criteria were patients with a definite DVT diagnosis who will receive an IVC filter to prevent fatal PE. As some indications are not absolute for IVC filter implantation, those who refused to insert an IVC filter were not enrolled. Patients were excluded if they (1) aged $<18$ or $>75$ years, (2) had obvious contraindications for anticoagulation therapy, (3) were allergic to iodine contrast agents, (4) had concomitant diseases requiring high-intensity anticoagulation and the anticoagulation intensity is higher than that of the patients with only an IVC filter, (5) had a creatinine clearance below $30 \mathrm{~mL} / \mathrm{min}$, (6) had clinically significant liver disease (eg, acute hepatitis, chronic active hepatitis or cirrhosis) or an alanine aminotransferase level that was more than three times the upper limit of the normal range, (7) had bacterial endocarditis, (8) had active bleeding or a potential bleeding risk, (9) were pregnant or breast-feeding, (10) had a systolic blood pressure of more than $180 \mathrm{~mm} \mathrm{Hg}$ or diastolic blood pressure of more than $110 \mathrm{~mm} \mathrm{Hg}$, (11) had malignant tumours and a life expectancy of $<1$ year or (12) taking CYP-450 3A4 inhibitors or inducers.

\section{Randomisation and blinding}

Blocked randomisation was performed. Central randomisation based on the Research Electronic Data Capture (REDCap) system will be conducted. The REDCap system was developed by Paul Harris of Vanderbilt University with a randomisation module. ${ }^{26}$ Someone not involved in this study will generate the allocation sequence using the REDCap system to ensure allocation concealment. Patients will be randomly assigned to either the experimental or the control group. It is an open-label study, and the assessors will be blinded to the interventions after the assignment.

\section{Therapy treatment and protocol}

The DVT patients will receive retrieval IVC filter implantation, the filters will be retrieved 1 month after insertion. In all participating centres, filters will be placed and retrieved by experienced vascular and interventional radiologists following a standardised procedure based on the technical documentation provided by the manufacturer. All patients will undergo cavography before and after filter placement and conventional abdominal radiography between 24 and 48 hours after implantation. Before filter retrieval, ultrasonography or venography will be performed to detect filter thrombosis.

Participants assigned to the rivaroxaban group will receive $20 \mathrm{mg}$ of rivaroxaban orally once daily for 4 months after the operation. Patients assigned to the standard therapy (ie, control) group will receive $1.0 \mathrm{mg} / \mathrm{kg}$ of body weight of nadroparin subcutaneously twice daily plus $3 \mathrm{mg}$ of warfarin orally once daily after the IVC filter insertion. Enoxaparin will be discontinued when the INR is between 2.0 and 3.0 or more for two consecutive days; the patient will receive at least 5 days of enoxaparin treatment.

\section{Discontinuaion or modification criteria}

Participants should withdraw from this clinical trial when they have active bleeding after anticoagulant administration, diseases that cannot continue anticoagulation or surgery is needed to stop anticoagulation. The detailed definitions for bleeding have been systematically described. ${ }^{27}{ }^{28}$ Subjects may be withdrawn from this study at their request or the request of their legal representative. However, early permanent discontinuation of the study drug is discouraged wherever possible and we will purchase insurance for all participants to cover studyrelated adverse events.

\section{Surveillance and follow-up}

All participating hospitals will use the same electronic case report form (eCRF) available in the REDCap system to collect patient data. All centres participating in the trial, ethics committee and statisticians will have access to the system, and patients' identity information is encrypted to prevent leakage. An independent data monitoring committee is responsible for summarising and checking the data of each centre. Moreover, if they have any questions, they can contact the coinvestigators of each centre for review or revision. We will follow the patients for the intended treatment period and assess them at the time of IVC filter retrieval (after 1 month) and 2 and 4 months after the operation in both study groups, using the eCRF data to elicit information on the symptoms and signs of recurrent VTE, bleeding and adverse events. Patients are instructed to report to the study centre immediately if any symptoms or signs suggestive of VTE or bleeding occurred between visits. 


\section{Outcome measures}

The primary outcomes of the study are death of any cause, PE-related death, bleeding and recurrent PE/ DVT. Patients with suspected recurrent PE/DVT and bleeding will undergo objective testing and a clinical summary form. Suspected recurrent PE/DVT is defined as a composite of DVT or non-fatal or fatal PE based on the previously described diagnostic criteria. ${ }^{29}$ Clinically relevant bleeding is defined as a composite of major or clinically relevant nonmajor bleeding, as also described previously. $^{30}$

The secondary outcomes are vascular events (eg, acute coronary syndrome, ischaemic stroke, transient ischaemic attack or systemic embolism), IVC filter retrieval failure and net clinical benefits (defined as a composite of the primary efficacy outcome and major bleeding assessed in the intention-to-treat population). IVC filter retrieval failure is relevant to IVC filter complications (eg, IVC thrombosis, IVC perforation, IVC filter migration or tilting and IVC filter embolisation), system factors and technical factors. ${ }^{31}$ We will also transform the retrieval IVC filters to permanent filters and prolong the duration of anticoagulant therapy. Bleeding will be defined as major if it is clinically overt and associated with a haemoglobin level decrease of $2.0 \mathrm{~g} / \mathrm{dL}$ or more, if bleeding leads to the transfusion of two or more units of red cells or if bleeding is intracranial or retroperitoneal, occurs in another critical site or contributes to death.

\section{Sample size calculation}

Adverse clinical outcomes of IVC filters are defined as a composite of recurrent VTE, IVC thrombosis or death. Weinberg et $a \hat{l}^{2}$ reported that the adverse clinical outcomes rate for patients with IVC filters and without anticoagulants was $67.9 \%$. However, the rate was only $30.5 \%$ and $29.1 \%$ for patients receiving prophylactic or therapeutic anticoagulation, respectively. For the group design non-inferiority test, 180 cases are sufficient under a power of 0.8 , and the non-inferiority margin was -0.12 . Thus, we will recruit 200 patients, considering a dropout rate of $10 \%, 100$ patients per group.

\section{Statistical analyses}

Statistical analyses will be performed on an intentionto-treat basis. Descriptive data will be reported as either means $\pm \mathrm{SD}$, medians $(\mathrm{IQR})$ or numbers and percentages. Abnormally distributed data will be presented as medians (IQRs). The $\chi^{2}$ will be used to compare categorical variables between the two groups. Continuous variables will be compared using the sample t-test for normally distributed data and the Mann-Whitney $U$ test for abnormally distributed continuous variables. The binary classification outcome variables will first be compared using a non-corrected $\chi^{2}$ test, and then the binary logistic regression analysis wil be established to correct for other confounding factors and calculate the ORs and 95\% CIs. The survival differences between the two groups will be compared using the log-rank test, and the results will be described by Kaplan-Meier survival curves. Statistical analyses will be performed by using GraphPad Prism (V.X, Microsoft, San Diego, CA, USA), and R software (R Foundation for Statistical Computing, Vienna, Austria). Statistical significance will be defined as a p-value of $<0.05$.

\section{Patient and public involvement}

Patients or the public were not involved in the study design, recruitment and conduct. The study results will be disseminated to study participants via a thank you letter at the end of the study.

\section{ETHICS AND DISSEMINATION \\ Approval and consent to participate}

The study was approved by the Human Research Ethics Committee of the Second Affiliated Hospital of Zhejiang University School of Medicine (approval number: (2019) 295). This trial was also approved by other centres' ethics committees, such as the Ethics Committee of Sir Run Run Shaw Hospital of Zhejiang University School of Medicine, the Ethics Committee of Zhongshan Hospital of Fudan University, the Ethics Committee of Huadong Hospital of Fudan University, the Ethics Committee of The Fifth People's Hospital of Shanghai, the Ethics Committee of Yantai Yuhuangding Hospital, the Ethics Committee of The First Affiliated Hospital of USTC, the Ethics Committee of Zhejiang Provincial People's Hospital, the Ethics Committee of Zhejiang Xiaoshan Hospital and the Ethics Committee of Hangzhou Third Hospital. An English translation of the ethical approval document is attached at online supplemental appendix 1. Written informed consent to participate (online supplemental appendix 2) will be obtained from all participants.

\section{Dissemination}

Study information will be prepared following the Good Clinical Practice Guidelines and is publicly available at www clinicaltrialsgov. The results of this trial will be submitted for publication in a peer-reviewed journal. The data will be used for publication and presentation at scientific meetings.

\section{DISCUSSION}

IVC filters are designed to trap venous emboli from the lower extremity and prevent clinically significant PE. The most common indication for IVC filter placement is an absolute contraindication to anticoagulation during the acute treatment of DVT or PE. The implantation of IVC filters for other indications, such as anticoagulation failure, massive clot burdens or reduced cardiopulmonary reserve, before thrombolysis or prophylaxis in highrisk patients has expanded. ${ }^{6}$ The availability of retrievable IVC filters has likely contributed to the increasing filter use. $^{25}$ Vena cava filters are thrombogenic, and thrombi were macroscopically detected in the removed temporary filters in $75 \%$ of patients after a mean insertion duration 
of only 5 days. ${ }^{33}$ Haemodynamic changes in the IVC after filter placement may increase the risk of thrombosis, ${ }^{8}$ and evidence suggests that IVC filters increase the DVT risk after filter placement. ${ }^{34} 35$ Therefore, high-intensity anticoagulation should be performed as soon as the IVC filter is placed to prevent filter-related thrombosis and recurrent DVT or PE.

VKAs administration after initial parenteral heparin treatment is recommended for long-term oral treatment in patients with an IVC filter. However, treatment with a VKA requires laboratory monitoring and dose adjustment and may be complicated by drug and food interactions, presenting a challenge to outpatient management. Novel oral anticoagulants (NOACs) are an ideal alternative because they do not require laboratory monitoring and have fewer drug, disease and diet interactions. ${ }^{1}$ However, there is no high-level evidence-based clinical verification for NOACs in anticoagulation regimens after implanting IVC filters. Therefore, it is necessary to conduct a randomised controlled trial to compare the anticoagulant effects of NOACs with warfarin. As a direct factor Xa inhibitor of NOACs, rivaroxaban has been demonstrated as effective and safe in the prophylaxis and treatment of VTE and as an antithrombotic therapy for atrial fibrillation, ${ }^{36}$ and peripheral or carotid artery disease. ${ }^{37}$ Therefore, rivaroxaban was chosen for this trial.

The study has some limitations. First, this is an openlabel study. Researchers and competent physicians are aware of the patients' grouping information, so we will arrange for independent coordinators blinded to the allocation of groups to collect the outcome data to reduce bias. Second, as the patients in the control group require INR and dose adjustment monitoring of warfarin during the follow-up period, they are not blinded to the treatment strategy, bringing more bias. However, we will make every effort to ensure that outcome assessors, data managers and statisticians are unaware of the treatment allocations. Third, different brands of retrieval IVC filters have their own advantages, limitations and complications rates. Owing to the variable medical insurance policies of participating centres, the obtained filters are different, which may also lead to errors in the results. We will use a single filter model to ensure study homogeneity, unify the indwelling time and reduce the corresponding errors.

Altogether, this trial aims to provide more clinical basis for anticoagulation after IVC filter implantation and explore the clinical indications for rivaroxaban.

\section{Trial status}

Ethics approval was granted before submission. Recruiting patients for the trial has not started (scheduled date: 31 December 2020). We anticipate that recruitment will be completed on 31 December 2021. The current protocol is V.4.0, and any protocol amendments will be updated at clinicaltrials.gov.

\section{Author affiliations}

${ }^{1}$ Vascular Surgery, Zhejiang University School of Medicine Second Affiliated Hospital, Hangzhou, Zhejiang, China

${ }^{2}$ Vascular Surgery, Zhejiang University School of Medicine Sir Run Run Shaw Hospital, Hangzhou, Zhejiang, China

${ }^{3}$ Vascular Surgery, Zhongshan Hospital Fudan University, Shanghai, China

${ }^{4}$ Vascular Surgery, Huadong Hospital Affiliated to Fudan University, Shanghai, China

${ }^{5}$ Vascular Surgery, Shanghai Fifth People's Hospital, Shanghai, China

${ }^{6}$ Vascular Surgery, Qindao University Medical College Affiliated Yantai Yuhuangding Hospital, Yantai, Shandong, China

${ }^{7}$ Vascular Surgery, Anhui Provincial Hospital, Hefei, Anhui, China

${ }^{8}$ Vascular Surgery, Zhejiang Provincial People's Hospital, Hangzhou, Zhejiang, China

${ }^{9}$ Vascualr Surgery, Zhejiang Xiaoshan Hospital, Hangzhou, Zhejiang, China

${ }^{10}$ Vascular Surgery, Third Peoplles Hospital of Hangzhou, Hangzhou, Zhejiang, China

${ }^{11}$ Department of Clinical Medicine Engineering, The Second Affiliated Hospital of

Zhejiang University, School of Medicine, Hangzhou, China

Acknowledgements The authors are grateful to the research assistants and coordinators at all participating sites.

Contributors LBZ and MML are joint first authors. ZJL, LBZ, YFZ, ZYS, WZ, BG, LBL, ZDF, GWY, WH and LJW were involved in the conception and design of the study. ZJL and LBZ obtained the ethical approval. MML contributed to the trial registration on the ClinicalTrial.gov website. LBZ drafted the manuscript. LY and XYK carried out literature search. JNY performed manuscript review. ZG, LY, ZJL contributed significantly in funding acquisition. All authors edited and revised the manuscript and approved the final version.

Funding This work was supported by Department of Science and Technology of Zhejiang Province (Grant No. LGF19H180019), Zhejiang University 2018 Zhejiang Provincial Department of Education General Research Project (Natural Science) (Grant No. Y201840845) and the Medical and Health Science and Technology Plan Project of Zhejiang Province (Grant No. 2019PY033)

Competing interests None declared.

Patient consent for publication Not required.

Provenance and peer review Not commissioned; externally peer reviewed.

Supplemental material This content has been supplied by the author(s). It has not been vetted by BMJ Publishing Group Limited (BMJ) and may not have been peer-reviewed. Any opinions or recommendations discussed are solely those of the author(s) and are not endorsed by BMJ. BMJ disclaims all liability and responsibility arising from any reliance placed on the content. Where the content includes any translated material, BMJ does not warrant the accuracy and reliability of the translations (including but not limited to local regulations, clinical guidelines, terminology, drug names and drug dosages), and is not responsible for any error and/or omissions arising from translation and adaptation or otherwise.

Open access This is an open access article distributed in accordance with the Creative Commons Attribution Non Commercial (CC BY-NC 4.0) license, which permits others to distribute, remix, adapt, build upon this work non-commercially, and license their derivative works on different terms, provided the original work is properly cited, appropriate credit is given, any changes made indicated, and the use is non-commercial. See: http://creativecommons.org/licenses/by-nc/4.0/.

ORCID iD

Zhenjie Liu http://orcid.org/0000-0002-7757-5916

\section{REFERENCES}

1 Tritschler T, Kraaijpoel N, Le Gal G, et al. Venous thromboembolism: advances in diagnosis and treatment. JAMA 2018;320:1583-94.

2 Tagalakis V, Patenaude V, Kahn SR, et al. Incidence of and mortality from venous thromboembolism in a real-world population: the Q-VTE study cohort. Am J Med 2013;126:832.e13-832.e21.

3 Kearon C, Akl EA, Ornelas J, et al. Antithrombotic therapy for VTe disease: chest guideline and expert panel report. Chest 2016;149:315-52.

4 Ho KM, Rao S, Honeybul S, et al. A multicenter trial of vena cava filters in severely injured patients. N Engl J Med 2019;381:328-37.

5 Ahmed O, Sheikh S, Tran P, et al. Inferior vena cava filter evaluation and management for the diagnostic radiologist: a comprehensive review including inferior vena cava Filter-Related complications and preserve trial filters. Can Assoc Radiol J 2019;70:367-82. 
6 Duffett L, Carrier M. Inferior vena cava filters. J Thromb Haemost 2017:15:3-12.

7 Lee S-H, Kim H-K, Hwang J-K, et al. Efficacy of Retrievable inferior vena cava filter placement in the prevention of pulmonary embolism during Catheter-Directed thrombectomy for proximal lower-extremity deep vein thrombosis. Ann Vasc Surg 2016;33:181-6.

8 Singer MA, Wang SL. Modeling blood flow in a tilted inferior vena cava filter: does tilt adversely affect hemodynamics? J Vasc Interv Radiol 2011;22:229-35.

9 Mahmood SS, Abtahian F, Fogerty AE, et al. Anticoagulation is associated with decreased inferior vena cava Filter-Related complications in patients with metastatic carcinoma. Am J Med 2017;130:77-82.

10 Jain A, Cifu AS. Antithrombotic therapy for venous thromboembolic disease. JAMA 2017:317:2008-9.

11 Schünemann $\mathrm{HJ}$, Cushman M, Burnett $\mathrm{AE}$, et al. American Society of hematology 2018 guidelines for management of venous thromboembolism: prophylaxis for hospitalized and nonhospitalized medical patients. Blood Adv 2018;2:3198-225.

12 Breddin HK, Hach-Wunderle V, Nakov R, et al. Effects of a lowmolecular-weight heparin on thrombus regression and recurrent thromboembolism in patients with deep-vein thrombosis. $N$ Engl $J$ Med 2001;344:626-31.

13 Bates SM, Rajasekhar A, Middeldorp S, et al. American Society of hematology 2018 guidelines for management of venous thromboembolism: venous thromboembolism in the context of pregnancy. Blood Adv 2018;2:3317-59.

14 Schulman S, Zondag M, Linkins L, et al. Recurrent venous thromboembolism in anticoagulated patients with cancer: management and short-term prognosis. $J$ Thromb Haemost 2015:13:1010-8.

15 Voigtlaender M, Langer F. Low-Molecular-Weight heparin in cancer patients: overview and indications. Hamostaseologie 2019:39:067-75.

16 Mismetti P, Laporte S, Pellerin O, et al. Effect of a Retrievable inferior vena cava filter plus anticoagulation vs anticoagulation alone on risk of recurrent pulmonary embolism: a randomized clinical trial. JAMA 2015;313:1627-35.

17 Witt DM, Clark NP, Kaatz S, et al. Guidance for the practical management of warfarin therapy in the treatment of venous thromboembolism. J Thromb Thrombolysis 2016;41:187-205.

18 Chen S-Y, Wu N, Gulseth M, et al. One-Year adherence to warfarin treatment for venous thromboembolism in high-risk patients and its association with long-term risk of recurrent events. J Manag Care Pharm 2013;19:291-301.

19 EINSTEIN Investigators, Bauersachs R, Berkowitz SD, et al. Oral rivaroxaban for symptomatic venous thromboembolism. N Engl J Med 2010;363:2499-510.

20 Lewis S, Glen J, Dawoud D, et al. Venous thromboembolism prophylaxis strategies for people undergoing elective total knee replacement: a systematic review and network meta-analysis. Lancet Haematol 2019;6:e530-9.

21 Eriksson BI, Kakkar AK, Turpie AGG, et al. Oral rivaroxaban for the prevention of symptomatic venous thromboembolism after elective hip and knee replacement. J Bone Joint Surg Br 2009;91:636-44.

22 EINSTEIN-PE Investigators, Büller HR, Prins MH, et al. Oral rivaroxaban for the treatment of symptomatic pulmonary embolism. N Engl J Med 2012;366:1287-97.
23 Ay C, Beyer-Westendorf J, Pabinger I. Treatment of cancerassociated venous thromboembolism in the age of direct oral anticoagulants. Ann Oncol 2019;30:897-907.

24 Prins $\mathrm{MH}$, Lensing AWA, Brighton TA, et al. Oral rivaroxaban versus enoxaparin with vitamin $\mathrm{K}$ antagonist for the treatment of symptomatic venous thromboembolism in patients with cancer (EINSTEIN-DVT and EINSTEIN-PE): a pooled subgroup analysis of two randomised controlled trials. Lancet Haematol 2014;1:e37-46.

25 Jaff MR, McMurtry MS, Archer SL, et al. Management of massive and submassive pulmonary embolism, iliofemoral deep vein thrombosis, and chronic thromboembolic pulmonary hypertension: a scientific statement from the American heart association. Circulation 2011;123:1788-830.

26 Harris PA, Taylor R, Thielke R, et al. Research electronic data capture (REDCap)--a metadata-driven methodology and workflow process for providing translational research informatics support. J Biomed Inform 2009;42:377-81.

27 Schulman S, Angerås U, Bergqvist D, et al. Definition of major bleeding in clinical investigations of antihemostatic medicinal products in surgical patients. J Thromb Haemost 2010;8:202-4.

28 Schulman S, Kearon C, Subcommittee on Control of Anticoagulation of the Scientific and Standardization Committee of the International Society on Thrombosis and Haemostasis. Definition of major bleeding in clinical investigations of antihemostatic medicinal products in non-surgical patients. $J$ Thromb Haemost 2005;3:692-4.

29 Spencer FA, Emery C, Lessard D, et al. The worcester venous thromboembolism study: a population-based study of the clinical epidemiology of venous thromboembolism. $J$ Gen Intern Med 2006;21:722-7.

30 van Gogh Investigators, Buller HR, Cohen AT, et al. Idraparinux versus standard therapy for venous thromboembolic disease. $N$ Engl J Med 2007;357:1094-104.

31 Crumley KD, Hyatt E, Kalva SP, et al. Factors affecting inferior vena cava filter retrieval: a review. Vasc Endovascular Surg 2019;53:224-9.

32 Weinberg I, Abtahian F, Debiasi R, et al. Effect of delayed inferior vena cava filter retrieval after early initiation of anticoagulation. $A m \mathrm{~J}$ Cardiol 2014;113:389-94.

33 Konishi H, Miyoshi T, Neki R, et al. Intrapartum temporary inferior vena cava filters are rarely indicated in pregnant women with deep venous thromboses. J Vasc Surg Venous Lymphat Disord 2015;3:370-5.

34 Sarosiek S, Crowther M, Sloan JM. Indications, complications, and management of inferior vena cava filters: the experience in 952 patients at an academic hospital with a level I trauma center. JAMA Intern Med 2013;173:513-7.

35 PREPIC Study Group. Eight-Year follow-up of patients with permanent vena cava filters in the prevention of pulmonary embolism: the PREPIC (prevention Du Risque d'Embolie Pulmonaire par interruption cave) randomized study. Circulation 2005;112:416-22.

36 Yasuda S, Kaikita K, Akao M, et al. Antithrombotic therapy for atrial fibrillation with stable coronary disease. N Engl J Med 2019;381:1103-13.

37 Anand SS, Bosch J, Eikelboom JW, et al. Rivaroxaban with or without aspirin in patients with stable peripheral or carotid artery disease: an international, randomised, double-blind, placebocontrolled trial. Lancet 2018;391:219-29. 